Ronald Bachmann, Philipp Jäger* und Robin Jessen

\title{
A Split Decision: Welche Auswirkungen hätte die Abschaffung des Ehegattensplittings auf das Arbeitsangebot und die Einkommensverteilung?
}

https://doi.org/10.1515/zfwp-2021-2052

\begin{abstract}
In this paper, we use a microsimulation model to examine the labor supply implications of switching from joint taxation ("Ehegattensplitting”) to individual taxation. We show that the switch to individual taxation would increase labor supply by more than half a million full-time equivalents. However, such a reform also leads to financial losses for some groups of the population, which should be taken into account by economic policy makers.
\end{abstract}

Keywords: Ehegattensplitting, Arbeitsangebot, Einkommensverteilung JEL-Codes: H24, J22, D31

\section{Einleitung}

Die europäische Bevölkerung altert schnell. Es wird erwartet, dass die Zahl der Personen im erwerbsfähigen Alter in den nächsten Jahrzehnten deutlich zurückgehen wird (Abbildung 1). Politische Entscheidungsträger in ganz Europa sind daher bestrebt, die Erwerbsquote zu erhöhen ${ }^{1}$, um die negativen Auswirkungen des demographischen Wandels auf die Rentensysteme und das langfristige Wirtschaftswachstum abzumildern.

1 Die Erwerbsquote ist der Anteil der Personen im erwerbsfähigen Alter, die arbeiten.

*Kontakt: Philipp Jäger: RWI - Leibniz-Institut für Wirtschaftsforschung e.V., Postfach 1030 54, 45030 Essen, E-Mail: Philipp.Jaeger@rwi-essen.de.

Ronald Bachmann: RWI - Leibniz-Institut für Wirtschaftsforschung e.V., Postfach 1030 54, 45030 Essen, E-Mail: Ronald.Bachmann@rwi-essen.de.

Robin Jessen: RWI - Leibniz-Institut für Wirtschaftsforschung e.V., Postfach 1030 54, 45030 Essen, E-Mail: Robin.Jessen@rwi-essen.de. 


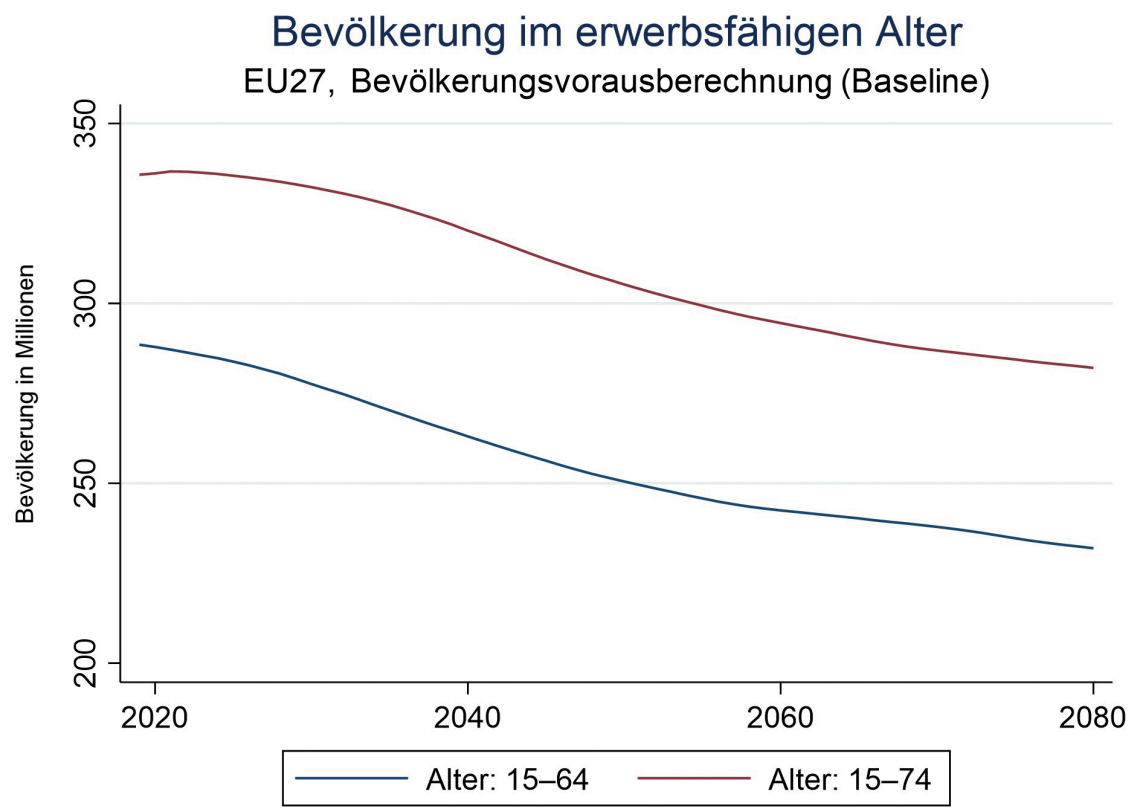

Abbildung 1: Die Alterung der Bevölkerung führt zu einem Rückgang der Zahl der Menschen im erwerbsfähigen Alter

Quelle: Eurostat (2021a).

Anmerkung: „Erwerbsalter“ in der engen (Alter 15-64) und weiten (Alter 15-74) Definition.

Insgesamt sind die Erwerbsquoten der Bevölkerung im erwerbsfähigen Alter in den EU27-Ländern seit 2000 gestiegen. Dieser Anstieg wurde hauptsächlich durch steigende Erwerbsquoten von Frauen getragen, wodurch sich zugleich die geschlechtsspezifischen Unterschiede bei der Beschäftigung verringert haben (Abbildung 2). Allerdings sind die Erwerbsquoten der Frauen immer noch mehr als 10 Prozentpunkte niedriger als die der Männer. Außerdem gehen Frauen häufiger einer Teilzeitbeschäftigung nach als Männer. Laut Eurostat arbeiteten im Jahr 2019 29,9 \% der Frauen in der EU27 in Teilzeit, verglichen mit 8,4\% der Männer. Die geschlechtsspezifischen Ungleichheiten auf dem Arbeitsmarkt könnten sich durch die jüngste Covid-19-Pandemie weiter verschärfen. Es gibt Hinweise darauf, dass zumindest in einigen entwickelten Volkswirtschaften Frauen stärker von der Pandemie betroffen waren. Hierfür gibt es zwei Gründe. Erstens arbeiten sie häufiger in stark betroffenen Sektoren. Und zweitens tragen sie oft einen höheren Anteil an Kinderbetreuungsaufgaben, wie z. B. Unterricht zu Hause; welche während der Pandemie zugenommen haben (Djankov et al. 2020). 


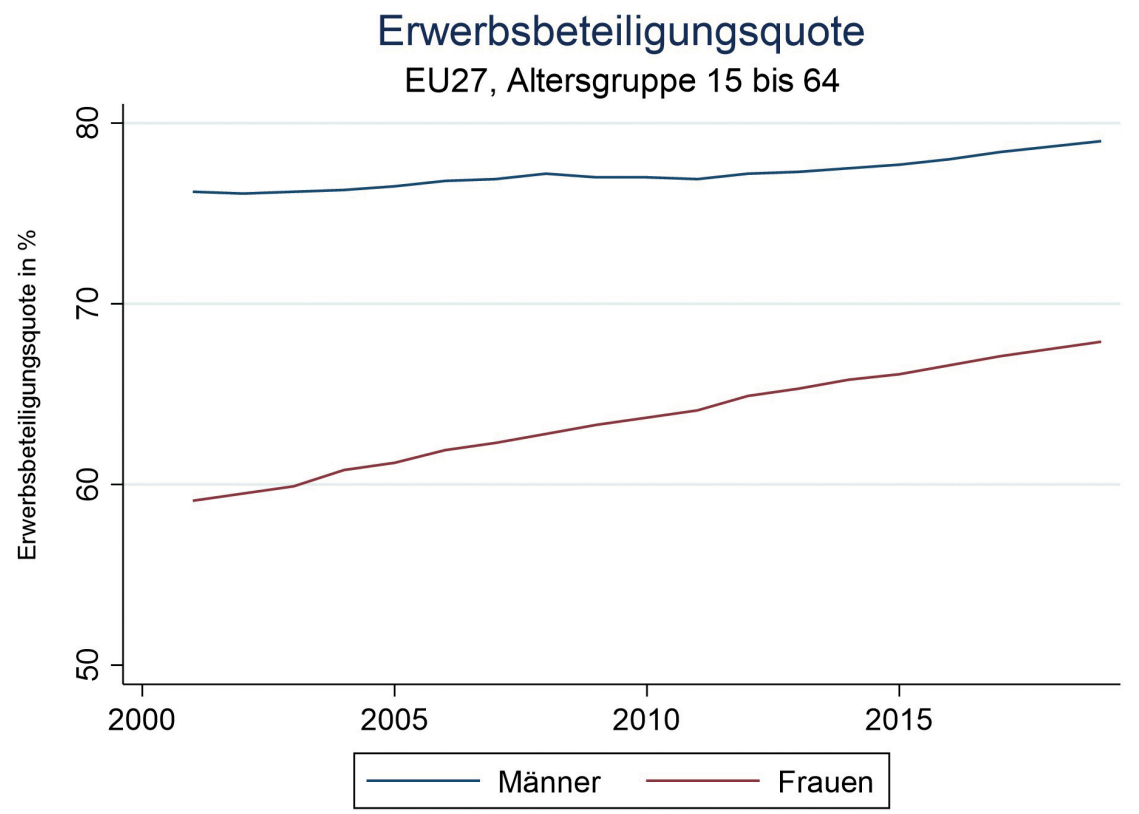

Abbildung 2: Die Frauenbeteiligung ist im Laufe der Zeit gestiegen, aber die Lücke bleibt bestehen Quelle: Eurostat (2021b).

Der geschlechtsspezifische Unterschied bei den Erwerbsquoten und den geleisteten Arbeitsstunden wirft die Frage auf, ob der Unterschied hauptsächlich auf persönliche Präferenzen oder auf politisch bedingte Fehlanreize zurückzuführen ist. Wenn letztere zumindest teilweise verantwortlich sind, könnte die Abschaffung dieser Fehlanreize die Erwerbsquoten insgesamt erhöhen und gleichzeitig zur Gleichstellung der Geschlechter beitragen.

Es gibt eine Reihe von Faktoren, die verhindern, dass sich die Partizipationslücke zwischen Frauen und Männern weiter verringert, wie z.B. der Mangel an Kinderbetreuungseinrichtungen (RWI2018). Zu den wichtigsten Faktoren gehören die europäischen Steuersysteme. Insbesondere die familienbasierte (d.h. gemeinsame) Besteuerung begünstigt (im Gegensatz zur Individualbesteuerung) Paare mit ungleichen Einkünften. Sie ist ein potenzielles Hindernis für die Erwerbstätigkeit von Frauen, da sie der Erwerbstätigkeit des Zweitverdieners (oft des weiblichen Ehepartners) negative Anreize setzt.

Diese Fehlanreize sind im deutschen Steuersystem besonders stark (OECD 2016). Daher werden in diesem Artikel sowohl der Arbeitsangebotseffekt als auch die Verteilungskonsequenzen eines Wechsels zur individuellen Einkommensbe- 
steuerung in Deutschland analysiert. Konkret untersuchen wir die Auswirkungen eines Wechsels von der gemeinsamen Besteuerung nach dem Splittingverfahren („Ehegattensplitting“), bei dem der Grenzsteuersatz ${ }^{2}$ für beide Ehepartner gleich ist und sich aus dem Durchschnittseinkommen des Paares ableitet, $\mathrm{zu}$ einer individuellen Besteuerung, bei der jeder Ehepartner einem eigenen Steuersatz unterliegt, der nur auf seinem eigenen Einkommen basiert. Um die Auswirkungen dieses Politikwechsels zu quantifizieren, wenden wir ein verhaltensbasiertes Mikrosimulationsmodell an. Dieses Modell bildet das deutsche Steuer- und Transfersystem detailliert ab und berücksichtigt, dass Menschen ihr Arbeitsangebot als Reaktion auf eine Politikänderung anpassen.

Unseren Simulationen zu Folge würde ein Wechsel zur Individualbesteuerung das Arbeitsangebot in Deutschland deutlich erhöhen. Der positive Effekt auf das Arbeitsangebot wäre noch größer, wenn die durch die Individualisierung der Besteuerung erhöhten Steuereinnahmen an die Ehepaare zurückgegeben würden. In unserer Simulation geschieht dies über eine Erhöhung des Grundfreibetrags bei der Einkommensteuer. In Deutschland wird das Einkommen nicht besteuert, wenn es unter dem Grundfreibetrag liegt (im Jahr 2021: 9.744€ für Alleinstehende und $19.488 €$ für Ehepaare $^{3}$ ). Die Umstellung auf die Individualbesteuerung und die Rückgabe der höheren Steuereinnahmen an Ehepaare würde das Arbeitsangebot um mehr als eine halbe Million zusätzlicher Vollzeitäquivalente (VZÄ) erhöhen. Da die simulierten Reformen Gewinner und Verlierer hervorbringen, ist letztlich ein Werturteil erforderlich, um zu entscheiden, ob die beträchtlichen Gewinne die Verluste für diejenigen überwiegen, die durch die Reform benachteiligt werden würden.

Unsere Studie leistet drei Hauptbeiträge zur Literatur. Erstens simulieren wir eine budgetneutrale Reform, bei der die Einführung der Individualbesteuerung durch das Anheben des Grundfreibetrags für verheiratete Paare begleitet wird. Zweitens zeigen wir Arbeitsangebotseffekte detailliert entlang der Einkommensverteilung. Drittens simulieren wir, im Unterschied $\mathrm{zu}$ vielen früheren Studien, Effekte auf die verfügbaren Einkommen entlang der Einkommensverteilung und für verschiedene Haushaltstypen.

Im nächsten Abschnitt geben wir einen Überblick über die wichtigsten Argumente für und gegen die Individualbesteuerung. Abschnitt 3 fasst kurz die bestehende Literatur zu den Arbeitsangebotseffekten der steuerlichen Individua-

2 Der Grenzsteuersatz ist der Steuersatz, der bei einer gegebenen Höhe des Einkommens auf einen zusätzlichen Euro des Einkommens angewendet würde, wenn das Einkommen um einen Euro erhöht werden würde.

3 Im Jahr 2015, dem Jahr, das wir unseren Simulationen zugrunde legen, lagen die Werte bei $8.472 €$ bzw. $16.944 €$. 
lisierung zusammen. Die Simulationen werden in Abschnitt 4 beschrieben und Abschnitt 5 enthält unsere Schlussfolgerungen.

\section{Argumente für und gegen das Ehegattensplitting}

Grundsätzlich können Ehepaare in Deutschland zwischen der gemeinsamen (Ehegattensplitting) und der individuellen Besteuerung wählen. Die Wahl der Individualbesteuerung führt jedoch nie dazu, dass sich ein Ehepaar finanziell besserstellt. Im Gegensatz dazu kann der Splittingvorteil beträchtlich sein, weil für die Berechnung des Durchschnittssteuersatzes das Durchschnittseinkommen der Ehegatten herangezogen wird, was oft zu einem deutlich niedrigeren Steuersatz führt als bei der Einzelveranlagung. Zum Beispiel kann ein Alleinverdiener-Ehepaar mit einem jährlichen zu versteuernden Einkommen von $60.000 €$ seine jährliche Steuerschuld gegenüber der Individualveranlagung um fast $6.000 €$ reduzieren (5.866 € basierend auf dem Steuertarif 2015). Bei gemeinsamer Besteuerung sinkt der durchschnittliche Einkommenssteuersatz eines solchen Paares von $28 \%$ auf $18 \%$. Verdienen die Ehegatten jedoch jeweils $30.000 €$, würden sie sowohl bei der Einzel- als auch bei der Zusammenveranlagung einem durchschnittlichen Steuersatz von $18 \%$ unterliegen. Daher profitieren Paare mit gleichem Einkommen nicht von der gemeinsamen Besteuerung.

Aufgrund der Progression des Steuersystems - der Grenzsteuersatz steigt mit dem Einkommen - ist die gemeinsame Besteuerung immer dann vorteilhaft, wenn die Einkünfte der Ehegatten unterschiedlich sind, das Paar Einkommensteuer zahlt und mindestens ein Ehegatte weniger als die oberste Grenzsteuergrenze verdient. Daher ist die gemeinsame Besteuerung de facto die Norm für Ehepaare.

Das Hauptargument für die Steuerindividualisierung ist eine höhere wirtschaftliche Effizienz, d. h. eine geringere Verzerrung durch die Einkommensteuer. Bei der gemeinsamen Besteuerung haben beide Ehepartner den gleichen Grenzsteuersatz. Der Wechsel zur Individualbesteuerung erhöht den Grenzsteuersatz für den besser verdienenden Ehepartner (typischerweise den Mann) und senkt den Grenzsteuersatz für den schlechter verdienenden Ehepartner (typischerweise die Frau). Wenn das Arbeitsangebot des Zweitverdieners stärker auf finanzielle Anreize reagiert als das des Hauptverdieners, ist die durch die gemeinsame Besteuerung induzierte Verzerrung der Arbeitsangebotsanreize größer als die durch die individuelle Besteuerung induzierte.

Da höhere Grenzsteuersätze den Arbeitsanreiz verringern, sollte die Abschaffung des Ehegattensplittings daher das Arbeitsangebot des besser verdienenden 
Ehepartners verringern und gleichzeitig das Arbeitsangebot des schlechter verdienenden Ehepartners erhöhen. Der geringer verdienende Ehepartner ist typischerweise die Frau, und es hat sich gezeigt, dass Frauen stärker auf steuerliche Anreize reagieren, d. h. eine höhere Arbeitsangebotselastizität in Bezug auf Löhne und Steuern aufweisen (Keane 2011). Gründe für die höheren Arbeitsangebotsreaktionen bei Frauen sind u.a. Präferenzen und die Rolle der nicht marktbestimmten Haushaltsproduktion (z. B. Kinderbetreuung). In diesem Fall sollte der Anstieg des Arbeitsangebots des Zweitverdieners den Rückgang des Arbeitsangebots des Erstverdieners mehr als kompensieren.

Ein weiteres Argument für die individuelle Besteuerung ist, dass die gemeinsame Besteuerung auch die Verteilung der Haushaltsressourcen negativ beeinflussen könnte. Die familienbasierte Besteuerung beruht auf der Idee, dass Paare ihre Ressourcen zusammenlegen. Empirische Studien haben jedoch gezeigt, dass es für die Verteilung der Haushaltsressourcen und die Verhandlungsmacht innerhalb des Haushalts darauf ankommt, wer das Geld tatsächlich verdient (Lundberg et al. 1997, Beznoska 2019). Zweitverdiener erhalten daher wahrscheinlich einen relativ kleinen Anteil des verdienten Geldes und werden somit nicht vollständig für ihre Arbeit im Haushalt entschädigt. Daher kann die Spezialisierung des Haushalts den Nebenverdienern schaden.

Das Hauptargument gegen die Individualbesteuerung ist die horizontale Gerechtigkeit, d.h. die Vorstellung, dass Paare mit dem gleichen Haushaltseinkommen den gleichen Steuerbetrag zahlen sollten. Das derzeitige Modell der gemeinsamen Besteuerung stellt sicher, dass Paare mit demselben Haushaltseinkommen denselben Steuerbetrag zahlen, unabhängig von der Verteilung des Haushaltseinkommens. Theoretisch garantiert die gemeinsame Besteuerung also (1), dass Paare entsprechend ihrer Fähigkeit, Steuern zu zahlen, besteuert werden, und (2) ermöglicht es dem Paar, frei zu entscheiden, wie es die Arbeits- und Nichtarbeitszeit innerhalb des Haushalts aufteilt. Darüber hinaus könnte die gemeinsame Besteuerung auch als Versicherung gegen den Verlust eines Arbeitsplatzes oder der Fähigkeit eines Ehepartners, ein Einkommen zu erzielen, dienen (Corneo 2013). Unter der Annahme, dass ein solches Risiko nicht versicherbar ist, könnte die gemeinsame Besteuerung der individuellen Besteuerung überlegen sein.

Tatsächlich waren Gerechtigkeitsbedenken der Hauptgrund für die Einführung des aktuellen gemeinsamen Besteuerungsmodells im Jahr 1958, nachdem das Bundesverfassungsgericht das bestehende Besteuerungsmodell als verfassungswidrig eingestuft hatte. Neuere Urteile des Bundesverfassungsgerichts, beginnend im Jahr 1982, könnten sogar implizieren, dass die Individualbesteuerung verfassungswidrig ist, weil sie den besonderen Charakter der Ehe, einschließlich der Verpflichtung, sich gegenseitig finanziell zu unterstützen, nicht berücksichtigt (Bundesministerium der Finanzen 2018). 
Das Argument, dass die gemeinsame Besteuerung die horizontale Gerechtigkeit sicherstellt, ist jedoch in die Kritik geraten. Bei einem festen Haushaltseinkommen ist ein Alleinverdienerhaushalt finanziell wohl bessergestellt als ein Doppelverdienerhaushalt, weil Ersterer mehr Zeit für Freizeit und Haushaltsproduktion (z. B. Putzen, Kochen und Kinderbetreuung) hat als Letztere (OECD 2016).

\section{Die in der Literatur diskutierten Effekte der Individualbesteuerung}

Bislang hat die ökonomische Literatur meist den Verdacht bestätigt, dass eine steuerliche Individualisierung die Effizienz steigert. Empirische Studien, die die Einführung der Steuerindividualisierung (oder Schritte in diese Richtung) in den USA, Kanada und Schweden untersuchten, legen nahe, dass das Arbeitsangebot von Frauen nach solchen Reformen tatsächlich zunahm (Eissa 1995; Crossley, Jeon 2007; Selin 2014). Analog dazu hatte der Wechsel von der individuellen zur gemeinsamen Besteuerung in den USA im Jahr 1948 den gegenteiligen Effekt (LaLumia 2008). Da Reformen zur Individualisierung der Steuern jedoch recht selten sind, bewerten die meisten Studien die Reformen nicht ex post, sondern verwenden theoretische Überlegungen in Kombination mit empirischen Daten, um die Auswirkungen politischer Reformen ex ante zu quantifizieren.

Zwei makroökonomische Studien kommen zu dem Schluss, dass die Erwerbsbeteiligung von Frauen in den USA erheblich steigen würde, wenn das Land zur Individualbesteuerung übergehen würde (Guner et al. 2012; Bick, Fuchs-Schündeln 2018). Aufgrund der unterschiedlichen Arbeitsangebots-elastizitäten zwischen Männern und Frauen führt die Individualbesteuerung auch insgesamt zu deutlich höheren Erwerbsquoten. Die Studie von Bick und Fuchs-Schündeln (2018) legt nahe, dass eine steuerliche Individualisierung in Deutschland einen noch größeren Effekt hätte, da das Steuersystem aufgrund der ausgeprägten Steuerprogression besonders starke Fehlanreize für Zweitverdiener bietet. Darüber hinaus zahlen Arbeitnehmer keine Einkommenssteuer auf Verdienste aus geringfügiger Beschäftigung, aus „Mini-Jobs“, die maximal $450 €$ pro Monat einbringen. Verdienen Zweitverdiener etwas mehr als dies, sinkt das Nettoeinkommen sogar, wie im nächsten Abschnitt näher erläutert wird. Die Kombination aus dieser Steuerbefreiung und der gemeinsamen Besteuerung wirkt sich negativ auf die Arbeitszeiten von Zweitverdienern aus (Steiner, Wrohlich 2005). Fehr et al (2015) bestätigt, dass die Einführung einer steuerlichen Individualisierung in Deutschland einen positiven Effekt auf die Effizienz hätte. 
Um die Auswirkungen der Steuerindividualisierung in Deutschland genauer zu beurteilen, werden Mikrosimulationsmodelle herangezogen. Frühere Studien, die solche Modelle verwenden (Bach et al. 1995; Bonin et al. 2013; Decoster, Haan, 2014; Steiner, Wrohlich 2004), legen nahe, dass die Steuerindividualisierung zu einem erheblichen Anstieg der Erwerbsbeteiligung von Frauen führen würde: zwischen zwei und fast fünf Prozentpunkten oder bis zu 11,4\% der geleisteten Arbeitsstunden. Außerdem übersteigt der Anstieg in allen Fällen die Verringerung des Arbeitsangebots von Männern, so dass es insgesamt zu einem Anstieg der Arbeitsstunden kommt. Da die steuerliche Individualisierung selbst die durchschnittlichen Steuersätze erhöht, würden auch die Staatseinnahmen steigen. Darüber hinaus simulieren die meisten der vorhandenen Studien Reformen, die den Staatshaushalt unverändert lassen. Sie erreichen die Haushaltsneutralität typischerweise durch pauschale Transfers, d. h. durch Geldtransfers, die für alle Steuerzahler gleich sind (Bach et al. 2017). Während Haushaltsneutralität über pauschale Transfers aus analytischer Sicht attraktiv ist, sind solche Reformszenarien nicht besonders realistisch. Die vorliegende Studie wählt stattdessen einen wohl realistischeren Ansatz und erreicht die Haushaltsneutralität durch eine Erhöhung des Grundfreibetrags für Ehepaare. Wie weiter unten beschrieben, hat dies den zusätzlichen Vorteil, dass die Grenzsteuersätze weiter sinken, was die Anreize für das Arbeitsangebot noch weiter erhöht.

Um die Ergebnisse vergleichbar zu machen, simulieren wir zunächst eine „reine“ Steuerindividualisierungsreform, die das Gesamtsteueraufkommen erhöht und damit das durchschnittlich verfügbare Haushaltseinkommen reduziert. In einem zweiten Schritt untersuchen wir eine haushaltsneutrale Reform, indem wir den jährlichen Grundfreibetrag und die Schwellenwerte aller Einkommenssteuerklassen anheben. Wir halten diese Reform für realistischer als die in der bestehenden Literatur häufig verwendeten pauschalen Transfers.

\section{Ergebnisse des Mikrosimulationsmodells}

\section{Beschreibung des Datensatzes und des Mikrosimulationsmodells}

Diese Studie verwendet das Sozio-oekonomische Panel (SOEP), eine jährliche repräsentative Befragung von mehr als 20.000 deutschen Haushalten (vgl. Wagner et al. 2007). Der Datensatz enthält detaillierte Informationen zu demographischen Merkmalen, Arbeitsangebot und Einkommen aus anderen Einkommensquellen. Die Studie verwendet retrospektive Daten für 2015. Seitdem hat es keine wesent- 
lichen Änderungen im deutschen Steuer-Transfer-System gegeben, so dass die Schlussfolgerung unserer Analyse weiterhin gültig ist.

Als Modellierungsrahmen verwendet die Studie das Einkommensteuer-Mikrosimulationsmodell EMSIM (Bechara et al. 2015) - Das Mikrosimulations-modell berechnet auf der Basis der aktuellen Regeln des Steuer-Transfer-Systems, der demographischen Merkmale und des Markteinkommens das verfügbare Einkommen für jeden Haushalt im SOEP. Das Simulationsmodell erfasst die wichtigen Details des Umverteilungssystems, einschließlich Steuern, Transfers, Sozialversicherungsbeiträge und Abzugsmöglichkeiten. Mit dem Modell lassen sich daher die Auswirkungen einer hypothetischen Reform simulieren.

Bei der Berechnung der Auswirkungen einer Reform berücksichtigt das Mikrosimulationsmodell, dass Individuen ihr Verhalten ändern können. Daher beinhaltet das Modell ein Arbeitsangebotsmodell, das im Anhang ausführlich beschrieben wird. Mit dem Mikrosimulationsmodell wird das verfügbare Einkommen berechnet, das den Haushalten bei unterschiedlicher Wahl der Arbeitszeit zur Verfügung stehen würde. Angesichts dieser Budgetbeschränkung entscheiden die Ehegatten gemeinsam über ihre Arbeitsstunden, um den Nutzen zu maximieren, der von Konsum und Freizeit beider Ehegatten abhängt. Eine Änderung des Steuersystems führt zu einer Änderung der Budgetbeschränkung des Haushalts. Die Haushalte könnten auf eine solche Änderung reagieren, indem sie ihr Arbeitsangebot anpassen.

Arbeitsangebotsreaktionen werden für Haushalte mit mindestens einem Ehepartner, der seine Arbeitszeiten ändern kann, simuliert. Dass das Arbeitsangebot flexibel ist, wird für alle Personen angenommen, mit Ausnahme derjenigen, die nicht im erwerbsfähigen Alter sind, derjenigen, für die in der Erhebung keine Angaben zur Arbeitszeit vorliegen, der Beamten, der Soldaten, der Selbstständigen, der Studenten, der Personen, die in geschützten Werkstätten arbeiten, und der Personen in Elternzeit. Die Arbeitsangebotsreaktionen dieser Gruppen werden nicht simuliert, entweder, weil die Personen in diesen Gruppen nicht frei sind, ihre Arbeitszeiten anzupassen, oder weil es Messprobleme gibt.

\section{Die Reformszenarien}

Im ersten Schritt simulieren wir die Auswirkungen eines Wechsels zur Individualbesteuerung ohne weitere Anpassungen des Steuersystems. Eine solche Reform erhöht die Steuerlast der meisten Ehepaare, und kein Haushalt ist unter diesem Szenario bessergestellt. Diese Simulation ermöglicht es jedoch, die Auswirkungen der Einführung der Individualbesteuerung von denen anderer Änderungen im Steuersystem abzugrenzen. 
Im zweiten Schritt simulieren wir die Auswirkungen einer Reform, die für den Staatshaushalt neutral ist. In diesem Szenario wird das erhöhte Steueraufkommen über eine Erhöhung des Grundfreibetrags, der nicht der Besteuerung unterliegt, an die Haushalte zurückgegeben. Unter der Annahme eines konstanten Arbeitsangebots kann der jährliche Grundfreibetrag für Ehepaare um $3.750 €$ erhöht werden (von 8.472€ auf 12.222 €). Unter Berücksichtigung des gestiegenen Arbeitsangebots könnte der Grundfreibetrag noch etwas stärker erhöht werden.

Es ist zu beachten, dass die Analyse nur für Ehepaare durchgeführt wird, da dies die einzige Gruppe ist, die direkt von einem Wechsel von der gemeinsamen zur individuellen Besteuerung betroffen ist. Der Grundfreibetrag wird nur für Ehepaare erhöht. Daher würden Alleinstehende und Ehepaare nach der Reform unterschiedlichen individuellen Steuersätzen unterliegen. ${ }^{4}$ Würde der Grundfreibetrag für alle Haushalte erhöht, wäre die mögliche Erhöhung deutlich geringer. Eine Reform mit einer geringeren Erhöhung des Grundfreibetrags würde Ehepaare deutlich schlechter stellen, während die meisten anderen Haushalte bessergestellt würden.

Alternative Wege zur Erreichung der Haushaltsneutralität wären ein pauschaler Transfer oder eine proportionale Senkung des Einkommensteuertarifs. Im Vergleich zu einem pauschalen Transfer bietet eine Erhöhung des Grundfreibetrags den Vorteil, dass sie für viele Haushalte zu einer Senkung der Grenzsteuersätze führt, was die Arbeitsangebotsanreize verbessert. Der Vorteil im Vergleich zu einer proportionalen Senkung der Einkommensteuersätze ist, dass eine solche Senkung vor allem Haushalten mit hohem Einkommen zugutekäme. Abbildung 3 zeigt einen Vergleich der Grenzsteuersätze der Einkommensteuer aus dem Jahr 2015 mit denen des Reformszenarios mit Haushaltsneutralität. In diesem Reformszenario sind die Grenzsteuersätze für ein zu versteuerndes Einkommen zwischen $8.472 €$ und $57.766 €$ niedriger als im Status quo.

\section{Vergleich von gemeinsamer und individueller Besteuerung}

Abbildung 4 zeigt, wie das verfügbare Einkommen von Ehepaaren ohne Kinder durch die Abschaffung des Ehegattensplittings beeinflusst wird. Dazu wird der jährliche Bruttoverdienst des einen Ehepartners (Person 2) auf $30.000 €$ fixiert, was in etwa dem durchschnittlichen Bruttoarbeitsverdienst in Deutschland entspricht. Der Verdienst des anderen Ehepartners (Person 1) variiert entlang der horizontalen Achse. Die durchgezogene Linie zeigt den Status quo mit gemeinsamer Besteuerung, die gestrichelte Linie zeigt das Szenario mit individueller

4 Das US-Steuersystem hat unterschiedliche Steuercodes für Einzelpersonen und Ehepaare. 


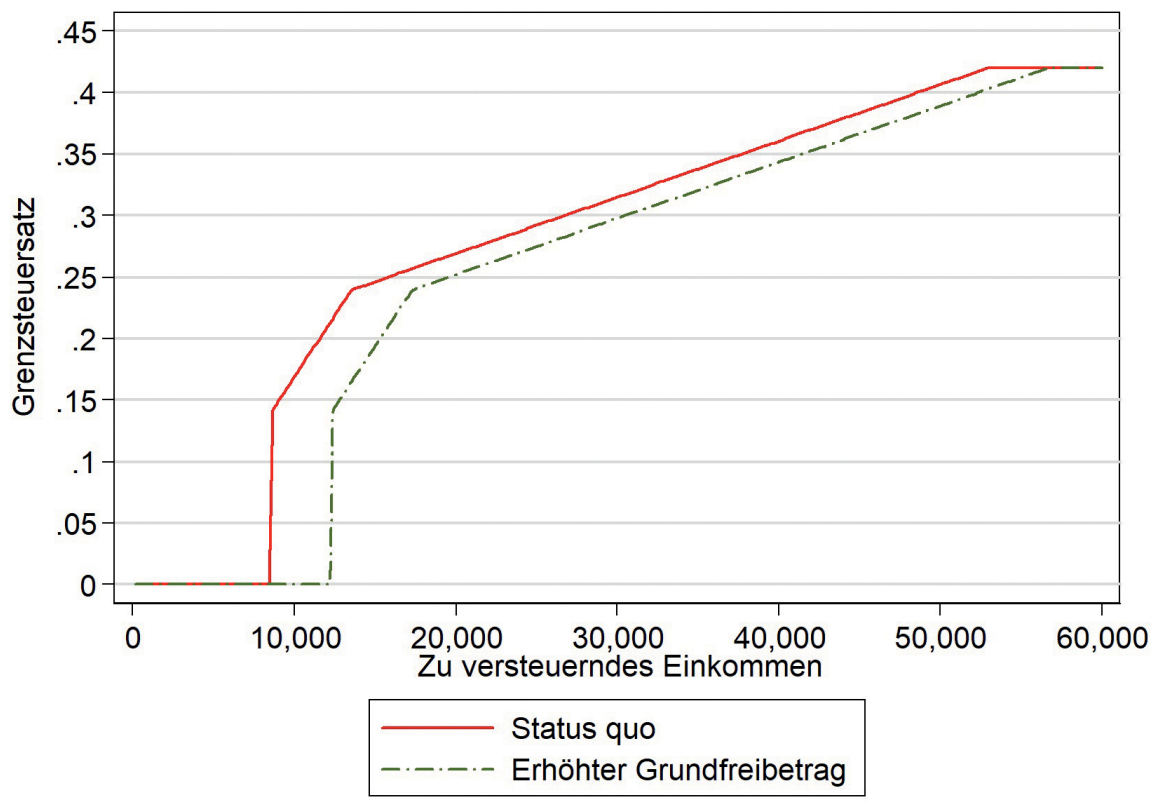

Abbildung 3: Der deutsche Einkommensteuertarif: Status quo und erhöhter Grundfreibetrag Quelle: Eigene Berechnung.

Besteuerung und die gestrichelt-gepunktete Linie zeigt das Szenario mit individueller Besteuerung und einer Verschiebung des Einkommensteuertarifs „nach rechts“, d. h. eine Erhöhung der Schwellenwerte aller Einkommenssteuerklassen. Erhöht sich das Bruttoarbeitseinkommen von $5.400 €$ auf $5.401 €$, führt dies zu einem Rückgang des verfügbaren Einkommens. Das liegt an der Schwelle für Minijobs. Monatsverdienste unterhalb der Minijobschwelle von $450 €$ sind aus Sicht des Arbeitnehmers komplett abgabenfrei, oberhalb der Schwelle wird die Einkommensteuer auf den gesamten Verdienst fällig. Die Kombination aus dieser Steuerbefreiung und der Zusammenveranlagung macht eine Aufstockung der Arbeitsstunden für Zweitverdiener unterhalb der Schwelle äußerst unattraktiv. ${ }^{5}$

Es können drei Beobachtungen gemacht werden. Erstens: Ein Wechsel zur Individualbesteuerung ohne Erhöhung des Grundfreibetrags verschlechtert die Situation der Haushalte, insbesondere derjenigen mit niedrigeren Einkommen. Wenn die Verdienste der beiden Ehepartner ähnlich hoch sind, hat der Wechsel

5 Bei der Individualbesteuerung gibt es noch einen leichten Rückgang des Nettoeinkommens an der Minijob-Grenze aufgrund der Sozialversicherungsbeiträge, aber dieser Rückgang ist geringer als der entsprechende Rückgang bei der gemeinsamen Besteuerung. 


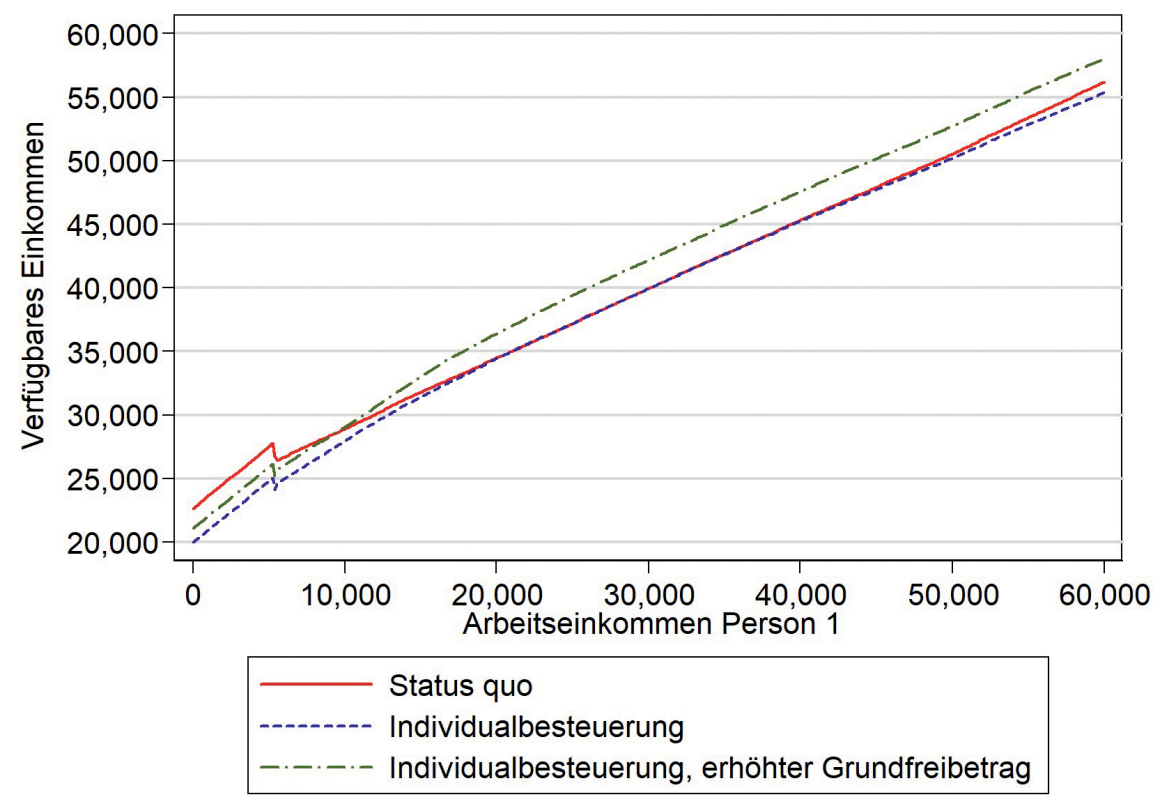

Abbildung 4: Verfügbares Haushaltseinkommen bei verschiedenen Verdienststufen für ein Ehepaar ohne Kinder; Verdienst von Person $2=30.000 €$ Quelle: Eigene Berechnung.

zur Individualbesteuerung nur einen sehr geringen Einfluss auf das verfügbare Einkommen. Zweitens: Bei relativ niedrigen Verdiensten (aber über $5.400 €$ ) ist die Linie bei der Individualbesteuerung steiler. Dies bedeutet, dass jeder zusätzliche Euro, den der Zweitverdiener verdient, zu einem größeren Einkommenszuwachs führt als unter dem Status quo. Es lohnt sich also unter der Individualbesteuerung eher für den Zweitverdiener, seine Arbeitszeit zu erhöhen. Drittens: Die Reform, die eine Erhöhung des Grundfreibetrags beinhaltet, stellt die Haushalte vieler Einkommensstufen besser und erhöht gleichzeitig die Arbeitsanreize. Tatsächlich ist die Budgetgerade im Vergleich zur „reinen“ Steuerindividualisierung ohne Erhöhung des Grundfreibetrags viel steiler.

Abbildung 4 zeigt auch, wie ein Wechsel einen Haushalt sogar besserstellen und gleichzeitig die Steuern, die der Haushalt zahlt, erhöhen kann. Nehmen wir an, dass die Zweitverdienerin einen Job hat, bei dem sie $10.000 €$ pro Jahr verdient. An diesem Punkt kreuzen sich die durchgezogene und die gestrichelt gezeichnete Linie. Wenn die individuelle Besteuerung mit einer Erhöhung des Grundfreibetrags eingeführt wird und sie den gleichen Job mit unveränderter Stundenzahl behält, bleibt das verfügbare Einkommen unverändert. Außerdem ändert sich 


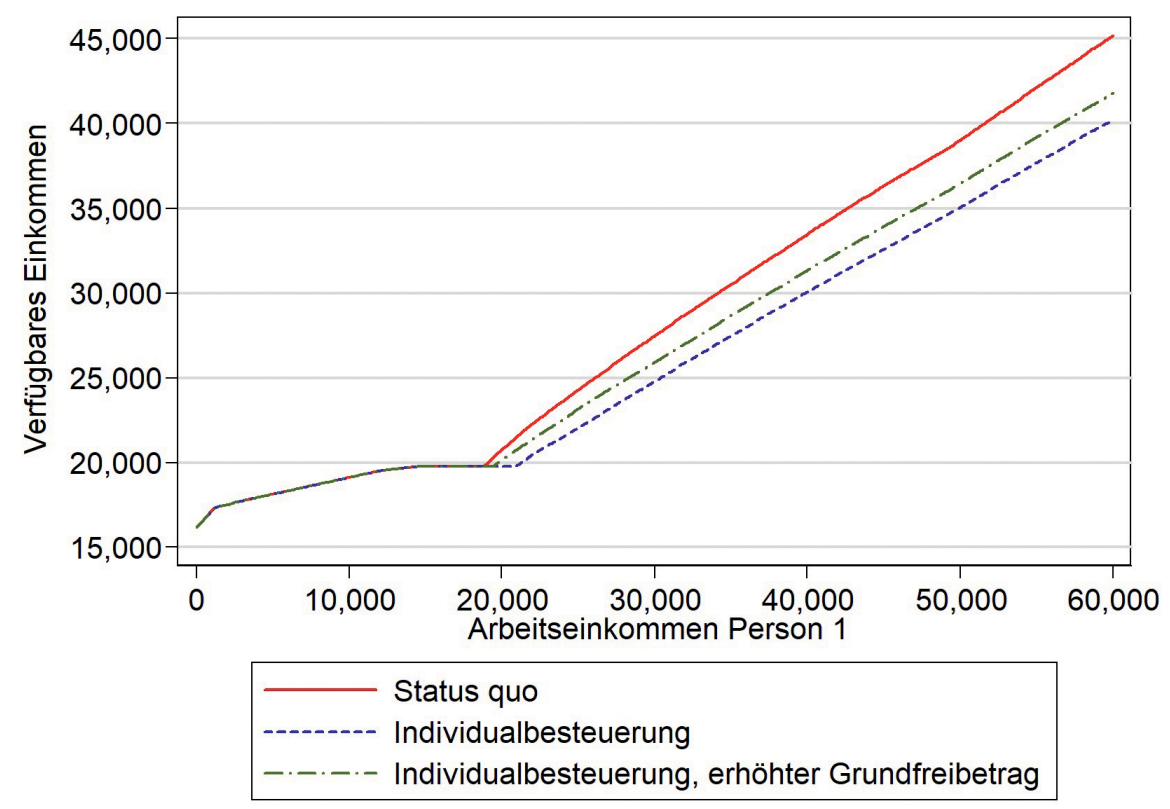

Abbildung 5: Verfügbares Haushaltseinkommen bei verschiedenen Verdienststufen für ein Ehepaar ohne Kinder; Verdienst von Person $2=5.000 €$ Quelle: Eigene Berechnung.

der Gesamtbetrag der Steuern und Sozialversicherungsbeiträge, den der Haushalt zahlt, nicht. ${ }^{6}$ Nehmen wir nun an, dass die Umstellung auf die Individualbesteuerung die Zweitverdienerin dazu veranlasst, ihre Arbeitszeit zu erhöhen, so dass sie $15.000 €$ pro Jahr verdient. Sie ist eindeutig bessergestellt, da ihr verfügbares Einkommen im Vergleich zum Status quo unverändert geblieben wäre, wenn sie weiterhin $10.000 €$ pro Jahr verdient hätte. Stattdessen hat sie sich entschieden, ihre Arbeitszeit zu erhöhen. Indem sie mehr arbeitet, zahlt sie auch mehr Steuern. Dieses Beispiel zeigt, dass die Reform zu Effizienzgewinnen führt, da sie die „Größe des Kuchens“ erhöht, der zwischen den Haushalten verteilt werden kann.

Abbildung 5 stellt die Situation eines Paares ohne Kinder dar, bei dem das Einkommen eines Ehepartners auf $5.000 €$ pro Jahr festgelegt ist. Hier ist der Effekt der Einführung der Individualbesteuerung ganz anders als im vorherigen Beispiel. Bei niedrigen Einkommensstufen hat eine Änderung der Einkommensbesteuerung keine Auswirkungen, da der Haushalt Anspruch auf Transferleistun-

6 Dies kann man erkennen, indem man das verfügbare Einkommen vom gesamten kombinierten Verdienst beider Ehegatten abzieht. 
gen hat. Bei höheren Einkommensniveaus verdient der Ehepartner, dessen Verdienst entlang der horizontalen Achse variiert, mehr als der andere Ehepartner. Nun sind die Linien in den Reformszenarien weniger steil als im Status quo. Die Grenzsteuersätze sind also höher und die Arbeitsanreize sind bei Individualbesteuerung niedriger als beim Ehegattensplitting.

\section{a. Arbeitsangebotseffekte}

Tabelle 1 zeigt die simulierten Arbeitsangebotseffekte eines Wechsels zur Individualbesteuerung und eines Wechsels zur Individualbesteuerung in Kombination mit einer Erhöhung des Grundfreibetrags der Einkommensteuer. Sie zeigt für beide Szenarien die prozentuale Veränderung der gesamten geleisteten Arbeitsstunden für verschiedene Haushaltstypen. Die Ergebnisse werden nur für die Haushalte angezeigt, bei denen mindestens ein Ehepartner die Arbeitsstunden anpassen kann (siehe Unterabschnitt 4.1).

Die Haushalte werden in Einkommensdezile eingeteilt, wobei Dezil 1 der niedrigsten und Dezil 10 der höchsten Einkommensgruppe entspricht. Unser Einkommensmaß ist das potenzielle Nettoäquivalenzeinkommen, d.h. das Nettoäquivalenzeinkommen, wenn beide Ehepartner mit flexiblem Arbeitsangebot Vollzeit arbeiten würden. Die Verwendung des potenziellen Einkommens ist insofern interessant, als dass es eher geboten erscheinen kann, an einen Haushalt umzuverteilen, der arm ist, weil das Verdienstpotenzial niedrig ist, als an einen Haushalt, der arm ist, weil die Ehepartner sich dafür entscheiden, weniger Stunden zu arbeiten. Um einen Vergleich von Haushalten unterschiedlicher Größe zu ermöglichen, werden die Nettoeinkommen anhand der modifizierten OECD-Skala in Nettoäquivalenzeinkommen umgerechnet. ${ }^{7}$ So ist ein Paar mit drei Kindern und einem Jahresnettoeinkommen von $40.000 €$ materiell schlechter gestellt als ein Paar ohne Kinder und demselben Jahresnettoeinkommen.

Die erste Zahl in Tabelle 1 zeigt, dass ein Übergang zur Individualbesteuerung Frauen im ersten Einkommensdezil insgesamt dazu veranlassen würde, ihre Arbeitsstunden im Vergleich zum Status quo um 0,3\% zu reduzieren. Die Arbeitsangebotseffekte im ersten Dezil sind gering, da diese Haushalte kaum Einkommensteuer zahlen. Die ersten drei Spalten zeigen, dass ein Wechsel zur Individualbesteuerung - vielleicht überraschend - die geleisteten Arbeitsstunden sowohl von Frauen als auch von Männern in den unteren vier Dezilen ver-

7 Dabei wird das Nettoeinkommen durch $(1+0,5 \times[$ Anzahl der Erwachsenen -1$]+0,3 \times[$ Anzahl der Kinder unter 14]) geteilt. 
mindern würde. Der Grund dafür ist, dass die Individualbesteuerung nur dann zu niedrigeren Grenzsteuersätzen für den weiblichen Ehepartner führt, wenn das Einkommen des anderen Ehepartners relativ hoch ist. Hat der männliche Ehepartner einen niedrigen Verdienst, führt die Individualbesteuerung zu einem Anstieg der Grenzsteuersätze für Frauen. Dies ist in Abbildung 5 dargestellt. Der negative Effekt auf das Arbeitsangebot der Männer folgt aus der Theorie: Für den Hauptverdiener führt die Individualbesteuerung zu höheren Grenzsteuersätzen.

Im Gegensatz dazu sind die positiven Arbeitsangebotsreaktionen der Frauen in den oberen sechs Dezilen erheblich. In den obersten drei Dezilen sind die Arbeitsangebotsanpassungen der Männer ebenfalls positiv. Dieser Effekt wird von männlichen Zweitverdienern getragen. Der Grenzsteuersatz von Hauptverdienern, deren Einkommen oberhalb der oberen Grenzsteuergrenze liegt, wird durch die Einführung der Individualbesteuerung nicht beeinflusst, so dass negative Arbeitsangebotsreaktionen in den oberen drei Dezilen begrenzt sind.

Der untere Teil der Tabelle zeigt, dass diese Steigerungen der durchschnittlich geleisteten Arbeitsstunden bei Paaren ohne Kinder am geringsten und bei Paaren mit mindestens zwei Kindern am größten sind. Der Grund dafür ist, dass der Unterschied im Verdienstniveau zwischen den Ehepartnern tendenziell höher ist, wenn Kinder im Haushalt vorhanden sind. In diesen Fällen verbessert die Individualbesteuerung die Arbeitsanreize der Zweitverdiener erheblich. Die letzte Zeile der Tabelle 1 zeigt die geschätzten Erhöhungen der geleisteten Arbeitsstunden in VZÄ, d.h. der aggregierte Anstieg der geleisteten Arbeitsstunden pro Woche wird durch 38 geteilt. ${ }^{8}$ Nach unserer Simulation erhöht die Einführung der Individualbesteuerung die Gesamtzahl der geleisteten Arbeitsstunden um etwa 389.000 VZÄ. Dieser Anstieg wird durch die steigenden Arbeitsstunden der Frauen getragen, während die Arbeitsstunden der Männer leicht sinken.

Die letzten drei Spalten in Tabelle 1 zeigen die Arbeitsangebotseffekte eines Wechsels zur Individualbesteuerung bei gleichzeitiger Erhöhung des Grundfreibetrags. Insgesamt negative Arbeitsangebotseffekte treten nur für das zweite bis dritte Dezil auf. Für die oberen sechs Dezile steigen die geleisteten Arbeitsstunden sowohl bei Frauen als auch bei Männern deutlich an. Die negativen Arbeitsangebotsanreize für Hauptverdiener und für Haushalte mit niedrigem Einkommen, die ein Wechsel zur Individualbesteuerung mit sich bringt, können also durch eine Erhöhung des Grundfreibetrags teilweise ausgeglichen werden.

Der untere Teil der Tabelle zeigt, dass für Paare mit und ohne Kinder die positiven Arbeitsangebotseffekte der Reform noch größer sind als die Effekte

8 In Tarifverträgen in Deutschland wird Vollzeit üblicherweise als 38 Stunden pro Woche definiert. Siehe Fuchs, et al. (2019). 
eines Wechsels zur Individualbesteuerung ohne Erhöhung des Grundfreibetrags. Der Grund dafür ist, dass die Erhöhung des Grundfreibetrags zu einer weiteren Senkung der Grenzsteuersätze führt, was die Arbeitsangebotsanreize weiter verbessert. Daher wäre der Gesamtanstieg der Arbeitsstunden bei dieser Reform noch größer: etwa 581.000 VZÄ. Auch dieser Anstieg würde hauptsächlich von Frauen getragen, aber auch Männer würden mehr Stunden arbeiten.

Tabelle 1: Auswirkungen eines Wechsels zur Individualbesteuerung auf die Gesamtzahl der geleisteten Arbeitsstunden, in Prozent der im Status quo geleisteten Arbeitsstunden

\begin{tabular}{|c|c|c|c|c|c|c|}
\hline & \multicolumn{3}{|c|}{ Individuelle Besteuerung } & \multicolumn{3}{|c|}{$\begin{array}{l}\text { Individuelle Besteuerung, } \\
\text { Erhöhung des Grundfreibetrags }\end{array}$} \\
\hline & Frauen & Männer & Gesamt & Frauen & Männer & Gesamt \\
\hline \multicolumn{7}{|l|}{ Dezile des potenziellen } \\
\hline \multicolumn{7}{|l|}{ Einkommens } \\
\hline 1. & -0.3 & -0.4 & -0.4 & 0.2 & -0.0 & 0.1 \\
\hline 2. & -3.8 & -3.9 & -3.8 & -1.5 & -1.6 & -1.6 \\
\hline 3. & -4.0 & -3.2 & -3.4 & -1.3 & -1.1 & -1.1 \\
\hline 4. & -1.6 & -3.8 & -3.0 & 3.4 & -0.8 & 0.7 \\
\hline 5. & 3.5 & -3.1 & -0.9 & 9.1 & -0.1 & 3.0 \\
\hline 6. & 5.5 & -1.4 & 1.1 & 10.2 & 0.6 & 4.1 \\
\hline 7. & 9.0 & -0.5 & 3.4 & 12.3 & 0.7 & 5.5 \\
\hline 8. & 9.1 & 0.3 & 4.0 & 11.5 & 0.7 & 5.2 \\
\hline 9. & 8.6 & 0.8 & 4.5 & 10.3 & 1.0 & 5.3 \\
\hline 10. & 9.2 & 0.8 & 4.6 & 10.4 & 0.7 & 5.1 \\
\hline \multicolumn{7}{|l|}{ Demografische Merkmale } \\
\hline Verheiratete Paare, 0 Kinder & 6.7 & 0.2 & 3.3 & 9.1 & 1.1 & 4.9 \\
\hline Verheiratete Paare, 1 Kind & 8.8 & -0.6 & 3.1 & 11.1 & 0.2 & 4.5 \\
\hline Verheiratete Paare, 2+ Kinder & 10.7 & -1.0 & 2.9 & 13.4 & -0.1 & 4.5 \\
\hline Alle Haushalte & 7.9 & -0.3 & 3.2 & 10.4 & 0.6 & 4.7 \\
\hline Effekt in VZÄ & 409,817 & $-21,177$ & 388,640 & 538,965 & 42,420 & 581,385 \\
\hline
\end{tabular}

Quelle: Eigene Simulation auf Basis des SOEP.

Anmerkung: Dezile des potenziellen Nettoäquivalenzeinkommens; Nettoäquivalenzeinkommen berechnet nach der modifizierten OCED-Skala, d. h. Haushaltseinkommen geteilt durch $(1+0,5 \times[$ Zahl der Erwachsenen - 1] + 0,3 $\times$ [Zahl der Kinder unter 14]). 
Der Vergleich der beiden Reformszenarien zeigt, dass ein erheblicher weiterer Anstieg der geleisteten Arbeitsstunden aus der Rückführung des Steuermehraufkommens resultiert, das durch einen Wechsel zur Individualbesteuerung über eine Erhöhung des Grundfreibetrags entsteht. Damit unterscheidet sich diese Reform deutlich von einer Reform mit pauschalem Transfer. Bei der letztgenannten Reform führen Einkommenseffekte zu niedrigeren Arbeitsanreizen.

Tabelle 2: Partizipationseffekte eines Wechsels zur Individualbesteuerung, in Prozentpunkten

\begin{tabular}{|c|c|c|c|c|c|c|}
\hline & \multicolumn{3}{|c|}{ Individuelle Besteuerung } & \multicolumn{3}{|c|}{$\begin{array}{l}\text { Individuelle Besteuerung, } \\
\text { Erhöhung des Grundfreibetrags }\end{array}$} \\
\hline & Frauen & Männer & Gesamt & Frauen & Männer & Gesamt \\
\hline \multicolumn{7}{|l|}{$\begin{array}{l}\text { Dezile des potenziellen } \\
\text { Einkommens }\end{array}$} \\
\hline 1. & 0.0 & -0.1 & -0.1 & 0.0 & 0.0 & 0.0 \\
\hline 2. & -0.3 & -1.0 & -1.0 & -0.1 & -0.4 & -0.4 \\
\hline 3. & -0.4 & -0.8 & -0.9 & -0.1 & -0.2 & -0.3 \\
\hline 4. & -0.4 & -1.1 & -1.3 & 0.4 & -0.2 & 0.0 \\
\hline 5. & 0.5 & -1.4 & -0.8 & 1.7 & 0.0 & 0.9 \\
\hline 6. & 1.5 & -0.7 & 0.4 & 2.5 & 0.4 & 1.8 \\
\hline 7. & 3.0 & -0.2 & 1.9 & 3.7 & 0.4 & 2.8 \\
\hline 8. & 3.4 & 0.2 & 2.5 & 3.8 & 0.5 & 3.0 \\
\hline 9. & 3.9 & 0.6 & 3.2 & 4.2 & 0.7 & 3.6 \\
\hline 10. & 4.2 & 0.6 & 3.4 & 4.5 & 0.6 & 3.6 \\
\hline \multicolumn{7}{|l|}{ Demografische Merkmale } \\
\hline Verheiratete Paare, 0 Kinder & 2.5 & 0.2 & 2.0 & 3.1 & 0.7 & 2.7 \\
\hline Verheiratete Paare, 1 Kind & 3.0 & -0.2 & 2.0 & 3.6 & 0.2 & 2.6 \\
\hline Verheiratete Paare, 2+ Kinder & 3.2 & -0.5 & 1.8 & 3.7 & 0.0 & 2.5 \\
\hline Alle Haushalte & 2.8 & 0.0 & 1.9 & 3.3 & 0.4 & 2.6 \\
\hline
\end{tabular}

Quelle: Eigene Simulation auf Basis des SOEP.

Anmerkung: Dezile des potenziellen Nettoäquivalenzeinkommens; Nettoäquivalenzeinkommen berechnet nach der modifizierten OCED-Skala, d. h. Haushaltseinkommen geteilt durch $(1+0,5$ $\times$ [Zahl der Erwachsenen - 1] + 0,3 × [Zahl der Kinder unter 14]. 
Tabelle 2 zeigt die Auswirkungen der beiden hypothetischen Reformen auf die Erwerbsbeteiligungsquoten in Prozentpunkten. So würde beispielsweise die Erwerbsquote der Männer im ersten Einkommensdezil um 0,1 Prozentpunkte sinken, wenn eine Individualbesteuerung eingeführt würde. In den meisten Fällen gehen positive Veränderungen der Erwerbsquote mit positiven Veränderungen der Gesamtarbeitszeit einher, die in Tabelle 1 dargestellt sind. Der größte Anstieg der Erwerbsquote würde für Frauen im zehnten Dezil eintreten. Ein Wechsel zur Individualbesteuerung in Verbindung mit einer Erhöhung des Grundfreibetrags würde ihre Erwerbsquote um 4,5 Prozentpunkte erhöhen. Bei den beiden Reformen würde der Gesamteffekt auf die Erwerbsquote aller verheirateten Personen mit flexiblem Arbeitsangebot 1,9 bzw. 2,6 Prozentpunkte betragen.

\section{b. Verteilungseffekte}

Wie das verfügbare Einkommen auf die beiden hypothetischen Reformen reagiert, hängt von der Position des Individuums in der Einkommensverteilung ab (Tabelle 3). ${ }^{9}$ Im Folgenden fassen wir die Verteilungseffekte der beiden Reformen zusammen, und zwar vor und nach der Anpassung des Arbeitsangebots:

- Der Übergang zur Individualbesteuerung allein (ohne Anpassung des Grundfreibetrags) hat negative Auswirkungen auf das verfügbare Einkommen von Ehepaaren über die gesamte Einkommensverteilung hinweg.

- Der Übergang zu einer haushaltsneutralen Individualbesteuerung mit Erhöhung des Grundfreibetrags führt dazu, dass das verfügbare Einkommen in den meisten Dezilen leicht sinken würde, wenn es keine Arbeitsangebotseffekte gäbe

- Nach Anpassung des Arbeitsangebots hat der Übergang zu einer haushaltsneutralen Individualbesteuerung positive Auswirkungen auf das durchschnittliche verfügbare Einkommen von Ehepaaren.

- Dieser Anstieg des verfügbaren Einkommens wird durch die obere Hälfte der Einkommensverteilung getrieben.

- Für die untere Hälfte der Einkommensverteilung sind die Auswirkungen auf das verfügbare Einkommen gering, bleiben aber negativ.

- Die Auswirkungen auf das verfügbare Einkommen unterscheiden sich nur geringfügig nach Familiengröße. Größere Familien weisen jedoch den geringsten Einkommenszuwachs nach Arbeitsangebotsanpassungen auf.

9 Es ist zu beachten, dass sich die Stichprobe in Tabelle 3 geringfügig von der in Tabelle 1 und 2 unterscheidet, da sie Paare enthält, die ihre Arbeitszeiten nicht anpassen können. 
Es ist zu beachten, dass eine Erhöhung des Nettoeinkommens nach Arbeitsangebotsanpassungen nicht zwangsläufig eine Erhöhung der Wohlfahrt impliziert, da höhere Einkommen durch längere Arbeitszeiten verursacht werden können und somit mit weniger Freizeit einhergehen. Andererseits impliziert ein höheres Nettoeinkommen vor der Anpassung des Arbeitsangebots notwendigerweise einen Anstieg der Wohlfahrt, da ein höheres Konsumniveau mit der gleichen Anzahl von Arbeitsstunden erreicht werden kann.

\section{c. Diskussion}

Die Simulation im vorangegangenen Unterkapitel hat gezeigt, dass die Einführung einer Individualbesteuerung die Gesamtzahl der geleisteten Arbeitsstunden aufgrund des höheren weiblichen Arbeitsangebots deutlich erhöhen würde. Eine solche Erhöhung des Arbeitsangebots würde zu einem einmaligen Anstieg des Bruttoinlandsprodukts führen.

Die Auswirkungen auf geleistete Arbeitsstunden könnten in der Realität etwas größer oder kleiner sein als in der Simulation. Zum einen haben wir nur eine partielle Gleichgewichtsanalyse durchgeführt und nicht berücksichtigt, dass eine Erhöhung des Arbeitsangebots die Marktlöhne senkt und dies wiederum die geleisteten Arbeitsstunden senkt. Somit könnten wir den positiven Arbeitsangebotseffekt überschätzt haben. Andererseits generiert eine Erhöhung des Arbeitsangebots zusätzliche Steuereinnahmen, die genutzt werden könnten, um die Anreize für das Arbeitsangebot noch weiter $\mathrm{zu}$ verbessern - beispielsweise durch eine weitere Erhöhung des Grundfreibetrags. Zudem erhöht ein steigendes Haushaltseinkommen typischerweise die Konsumausgaben, was das Wirtschaftswachstum ankurbeln könnte.

Ungeachtet dieser Vorbehalte hat die steuerliche Individualisierung einen erheblichen Vorteil. Die Reform verringert die Verzerrungen des Arbeitsangebots für Zweitverdiener, deren Arbeitsangebot typischerweise elastischer ist: Sie reagieren stärker auf finanzielle Anreize. Unter diesem Gesichtspunkt ist ein Wechsel zur Individualbesteuerung eindeutig wünschenswert, insbesondere wenn er mit einer Erhöhung der Einkommenssteuerfreibeträge kombiniert wird.

Allerdings schafft die Reform auch Verlierer und hat negative Verteilungsfolgen. Erstens sinkt das verfügbare Einkommen für die Mehrheit der Paare mit ungleichem Einkommen. Dies wirft Bedenken hinsichtlich der horizontalen Gerechtigkeit auf: Paare, die durch die Umstellung auf die Individualbesteuerung verlieren, tun dies nur, weil die Einkünfte nicht gleichmäßig zwischen den Ehegatten aufgeteilt werden. Im Gegensatz dazu hat die Einführung der Individualbesteuerung für Ehepaare mit gleichem Einkommen keine negativen Auswirkungen 


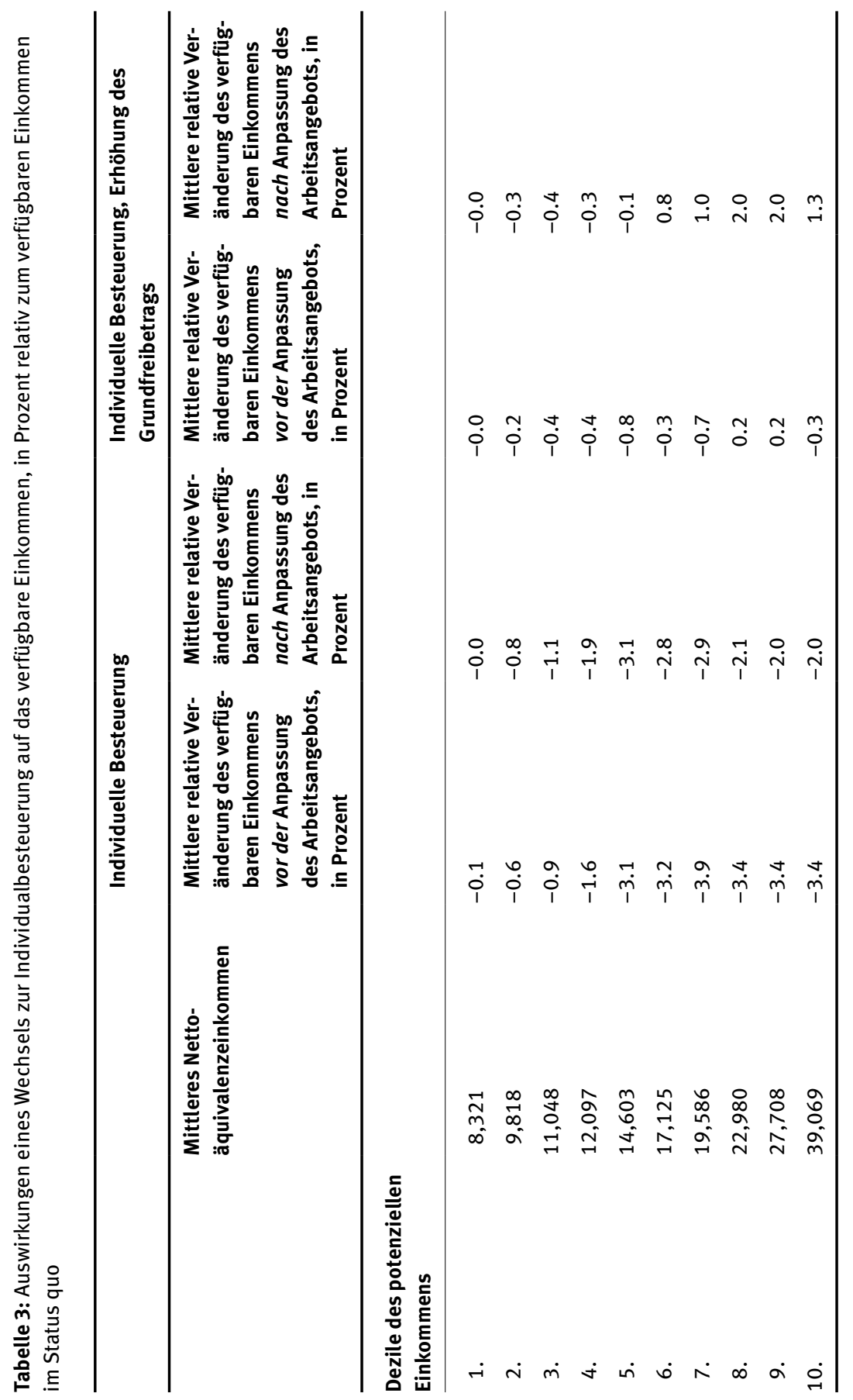




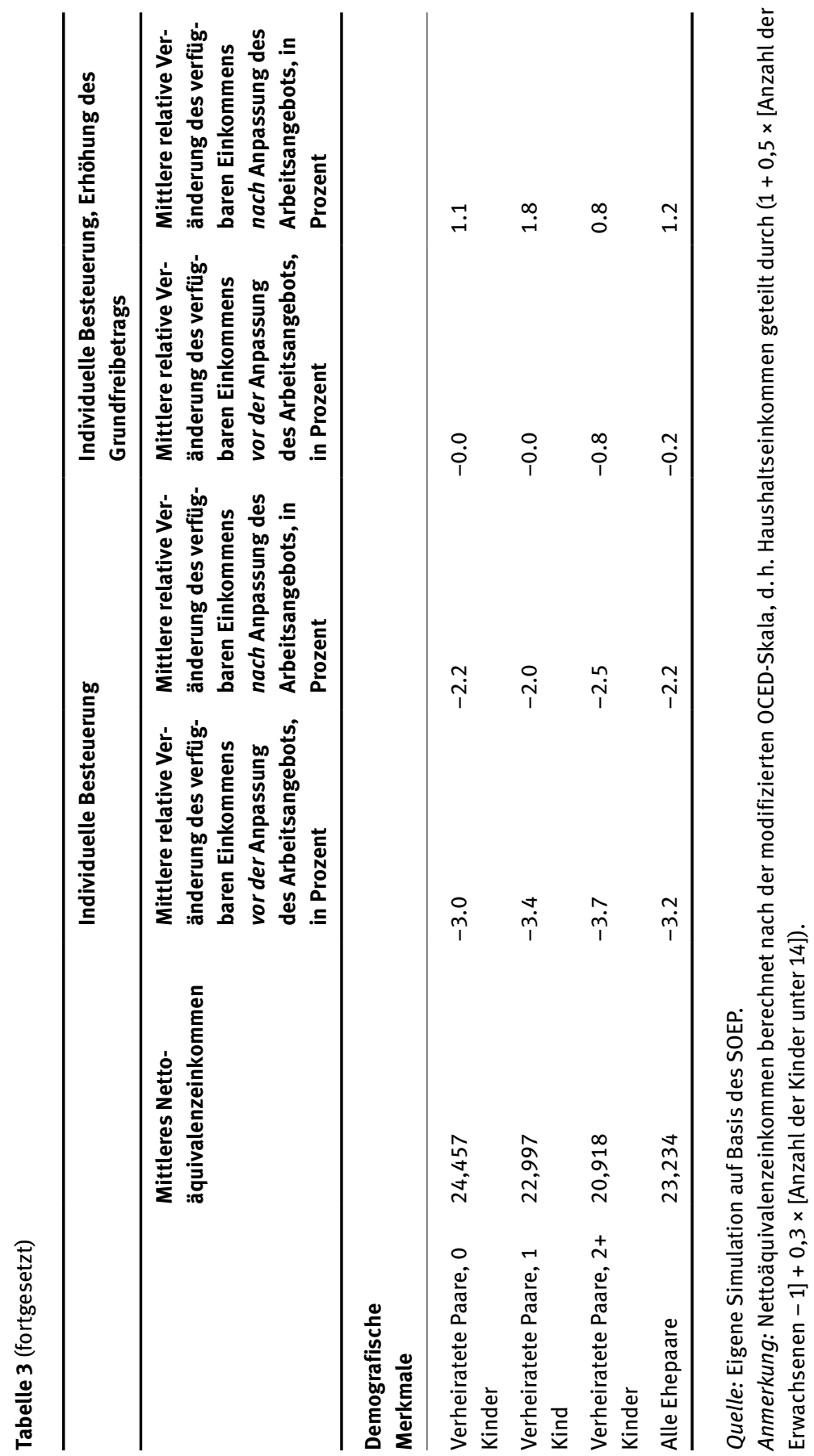


auf das verfügbare Einkommen. Aber wie oben argumentiert, ist dies nicht unbedingt ungerecht, da Doppelverdienerhaushalte oft höhere Kosten haben als Haushalte mit einem Alleinverdiener (z. B. Kinderbetreuung).

Zweitens sind die Verluste für Paare mit ungleichem Verdienst zwar relativ gering (bei der haushaltsneutralen Reform, nach Anpassung des Arbeitsangebots), aber sie konzentrieren sich auf finanziell gefährdete Gruppen: diejenigen am unteren Ende der Einkommensverteilung und Haushalte mit (zwei oder mehr) Kindern. Um die Bedenken hinsichtlich negativer Auswirkungen auf diese gefährdeten Gruppen zu verringern, könnte eine Umstellung auf die Individualbesteuerung z.B. mit folgenden zusätzlichen Maßnahmen einhergehen. Erstens könnten die Grenzentzugsraten von Transfers gesenkt werden; dies würde die Arbeitsanreize für einkommensschwache Haushalte verbessern und ihr verfügbares Einkommen erhöhen. Zweitens könnten das Kindergeld und der Kinderfreibetrag erhöht werden; dies würde zu einer zusätzlichen Senkung der Grenzsteuersätze für Eltern führen.

\section{Fazit}

Diese Studie hat gezeigt, dass ein Wechsel zur Individualbesteuerung das Arbeitsangebot in Deutschland deutlich erhöhen würde. Dieser Anstieg betrüge 389.000 VZÄ, wenn ein Wechsel zur Individualbesteuerung ohne zusätzliche Anpassung des Steuertarifs erfolgte. Würden die erhöhten Steuereinnahmen über eine Erhöhung des Grundfreibetrags an die Steuerzahler zurückgegeben, betrüge der Anstieg der geleisteten Arbeitsstunden 581.000 VZÄ. Berücksichtigt man allgemeine Gleichgewichtseffekte - z. B. niedrigere Löhne durch eine Erhöhung des Arbeitsangebots - könnten die Reformeffekte insgesamt etwas geringer ausfallen.

Angesichts der großen positiven Arbeitsangebotseffekte sind die aggregierten Gewinne größer als die aggregierten Verluste. Ein Wechsel zur Individualbesteuerung ist aus Effizienzgesichtspunkten eindeutig wünschenswert. Dennoch produziert eine solche Reform Gewinner und Verlierer, und es ist ein Werturteil erforderlich, um zu entscheiden, ob die Gewinne für einige die Verluste für andere überwiegen. Spezifische Maßnahmen, die auf die Verlierer eines Wechsels zur Individualbesteuerung abzielen, könnten eine solche Reform begleiten.

Die Ergebnisse dieser Studie wurden mit Daten aus der Zeit vor der Rezession 2020-21 aufgrund der Covid-19-Pandemie gewonnen. Nichtsdestotrotz bleiben die Schlussfolgerungen gültig: Eine Hinwendung zur Individualbesteuerung würde zusätzliche Steuereinnahmen generieren und das Arbeitsangebot erhöhen. Eine solche Steuerreform könnte ein Weg sein, die Verschuldung zu reduzieren und 
die Wirtschaft anzukurbeln. Sie könnte außerdem ein Weg sein, die Ungleichheiten zwischen den Geschlechtern auf dem Arbeitsmarkt zu mindern, die in einer Reihe von entwickelten Volkswirtschaften durch die Covid-19-Pandemie verschärft worden sind.

Anmerkung: Dieser Artikel ist eine übersetzte und gekürzte Version des Wilfried Martens Center for European Studies Research Papers „Me, Myself and I: Could Tax Individualisation Create Jobs and Reduce Inequality?“. Wir danken dem Wilfried Martens Center für finanzielle Unterstützung und Eoin Drea für hilfreiche Kommentare.

\section{Anhang: Technische Beschreibung des Arbeitsangebotsmodells}

Arbeitsangebotsreaktionen werden simuliert, indem für jeden Haushalt eine Nutzenfunktion geschätzt wird. Die Argumente dieser Nutzenfunktion sind das verfügbare Einkommen und die Freizeit des männlichen und weiblichen Ehepartners. Es wird angenommen, dass die Ehegatten gemeinsam ihren Nutzen maximieren, indem sie ihre Arbeitsstunden auf der Grundlage ihrer Budgetbeschränkungen wählen. Die Budgetbeschränkungen hängen vom Nicht-Arbeitseinkommen, dem Stundenlohn jedes Ehepartners und dem Steuer-Transfer-System ab. Die Wahrscheinlichkeit, eine bestimmte Anzahl von Stunden zu arbeiten, hängt von dieser geschätzten Nutzenfunktion ab. Wenn es eine Änderung des verfügbaren Einkommens gibt, das mit einer bestimmten Wahl der Stundenzahl verbunden ist, wie in unserem Fall durch die Einführung der Individualbesteuerung, ändern sich diese Wahrscheinlichkeiten.

Es wird angenommen, dass die Haushalte eine diskrete Auswahl an Arbeitsstunden pro Woche treffen. Frauen wählen zwischen 0, 10, 20, 39 und 45 Stunden und Männer zwischen 0, 20, 40 und 48 Stunden pro Woche. Somit wählen Haushalte mit zwei Ehepartnern mit flexiblem Arbeitsangebot zwischen 20 möglichen Kombinationen von Arbeitsstunden. Ein wesentlicher Vorteil dieses Ansatzes ist, dass das verfügbare Einkommen, das mit unterschiedlichen Arbeitszeitwahlen verbunden ist, mikrosimuliert werden kann, wobei die Details des deutschen Verteilungssystems berücksichtigt werden. $\mathrm{Zu}$ diesem Zweck verwenden wir das Mikrosimulationsmodell EMSIM. Die Informationen über die tatsächliche Arbeitszeit und das Bruttoeinkommen werden direkt aus dem SOEP entnommen, und die Stundenlöhne werden mit diesen Informationen berechnet. Für Arbeitslose werden potenzielle Stundenlöhne mit Hilfe einer selektivitätskorrigierten 
(Heckman 1979) Lohnregression auf Variablen, die sich auf das Humankapital beziehen, imputiert. Dies wird für Frauen und Männer in West- und Ostdeutschland getrennt durchgeführt. Die Nutzenfunktion wird dann unter Verwendung des Steuer- und Transfersystems und der Stundenwahl eines bestimmten Jahres geschätzt.

Die Schätzung der Arbeitsangebotsreaktionen folgt zwei Studien aus dem Jahr 1995 (Aaberge et al. 1995; Van Soest 1995). Die Anzahl der Freizeitstunden pro Woche wird mit lf für den weiblichen Ehepartner und durch lm für den männlichen Ehegatten bezeichnet. Der Haushaltskonsum - der in diesem Ein-Perioden-Modell dem verfügbaren Einkommen entspricht - wird mit $c$ notiert. Unter Ausschluss der haushaltsspezifischen Indizes ist die Nutzenfunktion eines Haushalts für eine gegebene Wahl der Arbeitsstunden $j$ ist gegeben durch

$V_{j}=U\left(l f_{j}, l m_{j}, c_{j}\right)+\epsilon_{j}$.

Die Nutzenfunktion besteht aus einem deterministischen Teil, $U$, und einer Zufallskomponente $\epsilon_{j}$. Der deterministische Teil hat die Form

$$
U=\beta_{1} \ln \left(c_{j}\right)+\beta_{2} \ln \left(c_{j}\right)^{2}+\beta_{3} \ln \left(l f_{j}\right)+\beta_{4} \ln (\operatorname{lm} j)+\beta_{5} \ln (\operatorname{lm} j) \ln \left(l f_{j}\right)+I_{j},
$$

wobei „ln“ den natürlichen Logarithmus bezeichnet. $I_{j}$ ist ein fixer Effekt, der für jede Stundenkategorie spezifisch ist und z. B. die Tatsache erfasst, dass Teilzeitarbeit in vielen Berufen unüblich ist. Wie in strukturellen Arbeitsangebotsmodellen mit diskreter Wahl üblich, wird Heterogenität zwischen den Haushalten eingeführt, indem die Koeffizienten der Nutzenfunktion von soziodemografischen Merkmalen wie Alter, Bildung und Anzahl der Kinder im Haushalt abhängen. Zum Beispiel wird die Freizeit des weiblichen Ehepartners von Paaren mit Kindern höher bewertet. Es wird angenommen, dass der Fehlerterm der Gumbel-Verteilung folgt. Die Wahrscheinlichkeit, dass ein bestimmter Haushalt die Arbeitszeitkategorie $j$ wählt, ist dann gegeben durch (McFaden 1974)

$$
P_{j}=\operatorname{Pr}\left(V_{j}>V_{i}, \forall i \neq j\right)=\frac{\exp \left(U_{j}\right)}{\sum_{i=1}^{I} \exp \left(U_{i}\right)} \text {. }
$$

Änderungen des Arbeitsangebots werden simuliert, indem das verfügbare Einkommen, das mit bestimmten Stundenwahlen verbunden ist, verändert wird, z. B. durch die Einführung einer individuellen Besteuerung. Dies führt zu einer Änderung des deterministischen Teils der Nutzenfunktion und damit der Wahlwahrscheinlichkeit.

Für diese Studie wird das Modell nur für verheiratete Paare geschätzt. Die Schätzung unterscheidet zwischen Haushalten mit zwei Ehepartnern mit flexib- 
lem Arbeitsangebot und solchen, bei denen nur ein Ehepartner die Arbeitsstunden ändern kann.

Tabelle 4 zeigt, um wie viel die geleisteten Arbeitsstunden von verheirateten Frauen und Männern steigen würden, wenn alle Stundenlöhne um 1\% erhöht würden. Diese Erhöhungen wurden simuliert, indem die Bruttolöhne von Frauen und Männern um 1\% erhöht wurden und dann die Reaktion des Arbeitsangebots simuliert wurde. Die Tabelle zeigt, dass bei Frauen eine Erhöhung der Bruttolöhne um 1\% zu einem Anstieg der geleisteten Arbeitsstunden um 0,47\% führt. Für Männer ist dieser Wert geringer (0,17\%). Diese Elastizitäten stehen im Einklang mit den gängigen Erkenntnissen in der Literatur zum Arbeitsangebot (Keane 2011). Die Feststellung, dass die geleisteten Arbeitsstunden von Frauen tendenziell stärker auf eine Lohnänderung reagieren, ist sehr verbreitet. Die zweite Zeile in der Tabelle zeigt, dass ein Anstieg der Bruttostundenlöhne um 1\% zu einem Anstieg der Erwerbsquote bei Frauen um 0,14 Prozentpunkte und bei Männern um 0,08 Prozentpunkte führen würde. Auch diese Zahlen sind plausibel und stehen im Einklang mit der vorhandenen Literatur.

Tabelle 4: Simulierter Anstieg der geleisteten Arbeitsstunden und der Teilnahmequoten aufgrund einer Erhöhung des Stundenlohnsatzes um 1,0\%

\begin{tabular}{lll}
\hline & Frauen & Männer \\
\hline Aggregierter Stundenzuwachs in Prozent & 0.47 & 0.17 \\
Anstieg der Beteiligungsquote in Prozentpunkten & 0.14 & 0.08 \\
\hline
\end{tabular}

Anmerkung: Simuliert mit einem Anstieg der Bruttolöhne um $1 \%$.

\section{Literatur}

Aaberge, R., Dagsvik, J. and Strøm, S., 'Labor Supply Responses and Welfare Effects of Tax Reforms', Scandinavian Journal of Economics 97/4 (1995), 635-59.

Bach, S. et al., 'Ehegattenbesteuerung: Individualbesteuerung mit übertragbarem Grundfreibetrag schafft fiskalische Spielräume', DIW-Wochenbericht 84 (2017), 247-55.

Bach, S. et al., 'Reform of Income Splitting for Married Couples: Only Individual Taxation Significantly Increases Working Incentives', DIW Economic Bulletin 5 (1995), 13-19.

Bechara, P., Kasten, T. and Schaffner, S., 'Dokumentation des RWI-Einkommensteuer-Mikrosimulationsmodells (EMSIM)', RWI Materialien 86 (2015).

Beznoska, M., Do Couples Pool Their Income? Evidence From Demand System Estimation for Germany, Discussion Papers 2019/3, Free University Berlin, School of Business \& Economics (2019). 
Bonin, H. et al., 'Evaluation zentraler ehe- und familienbezogener Leistungen in Deutschland Endbericht', Gutachten für die Prognos AG, Zentrum für Europäische Wirtschaftsforschung (Mannheim, 2013).

Bick, A. and Fuchs-Schündeln, N., 'Quantifying the Disincentive Effects of Joint Taxation on Married Women's Labor Supply', American Economic Review Papers \& Proceedings 107/5 (2017), 100-4.

Bundesministerium der Finanzen (2018) (Hrsg.), Wissenschaftlicher Beirat des Bundesfinanzministeriums, Gutachten zur Reform der Besteuerung von Ehegatten (2018).

Corneo, G., 'A Note on the Taxation of Couples Under Income Uncertainty', FinanzArchiv 69/1 (2013), 129-34.

Crossley, T. and Jeon, S.-H., 'Joint Taxation and the Labor Supply of Married Women: Evidence from the Canadian Tax Reform of 1988', Fiscal Studies 28 (2007), 343-65.

Decoster, A. and Haan, P., 'Welfare Effects of a Shift of Joint to Individual Taxation in the German Personal Income Tax', FinanzArchiv 70 (2014), 599-624.

Djankov, S., Trubmic, T. and Zhang, E., 'The Gender Gap and COVID-19: Evidence From Eight Advanced Economies', VoxEU.org, 14 December 2020, accessed at https://voxeu.org/ article/covid-19-and-gender-gap-advanced-economies on 11 February 2021.

Eissa, N. O., Taxation and Labor Supply of Married Women: The Tax Reform Act of 1986 as a Natural Experiment, NBER Working Paper 5023 (1995).

Eurostat, 'Employment and Activity by Sex and Age: Annual Data’, [lfsi_emp_a] (2021a), accessed at https://appsso.eurostat.ec.europa.eu/nui/show.do?dataset=lfsi_emp_ a\&lang=en on 4 February 2021.

Eurostat, 'Part-Time Employment and Temporary Contracts: Annual Data', [lfsi_pt_a] (2021b), accessed at https://appsso.eurostat.ec.europa.eu/nui/show.do?dataset=Ifsi_ pt_a\&lang=en on 4 February 2021.

Eurostat, 'Population on $1^{\text {st }}$ January by Age, Sex and Type of Projection', [proj_19np] (2021), accessed at https://appsso.eurostat.ec.europa.eu/nui/show.do?dataset=proj_19np\& lang=en on 4 February 2021.

Fehr, H., Kallweit, M. and Kindermann, F., 'Reforming Family Taxation in Germany - Labor Supply Versus Insurance Effects', FinanzArchiv 71 (2015), 53-81.

Fuchs, J. et al., IAB-Prognose 2019: Trotz Konjunkturflaute: Arbeitsmarkt hält Kurs, IAB-Kurzbericht 7 (Nürnberg, 2019).

Guner, N., Kaygusuz, R. and Ventura, G., 'Taxation and Household Labor Supply', Review of Economic Studies 79 (2012), 1113-49.

Heckman, J., 'Sample Selection Bias as a Specification Error', Econometrica 47/1 (1979), 153-61.

Keane, M. P., 'Labor Supply and Taxes: A Survey', Journal of Economic Literature 49/4 (2011), 961-1075.

LaLumia, S., 'The Effects of Joint Taxation of Married Couples on Labor Supply and Non-Wage Income', Journal of Public Economics 92 (2008), 1698-1719.

Lundberg, S. J., Pollak, R. A. and Wales, T. J., 'Do Husbands and Wives Pool Their Resources? Evidence From the United Kingdom Child Benefit', Journal of Human Resources 32/3 (1997), 463-80.

McFadden, D., 'Conditional Logit Analysis of Qualitative Choice Behavior', in P. Zarembka (ed.), Frontiers in Econometrics (New York: Academic Press, 1974), 105-142.

OECD, Taxing Wages 2014-2015 - Special Feature: Measuring the Tax Wedge on Second Earners (Paris, 2016). 
RWI, Working Women and Labour Market Inequality, RWI Projektberichte (2018).

Scientific Advisory Board of the German Ministry of Finance, Gutachten zur Reform der Besteuerung von Ehegatten (2018).

Selin, H., 'The Rise in Female Employment and the Role of Tax Incentives: An Empirical Analysis of the Swedish Individual Tax Reform of 1971', International Tax and Public Finance 21 (2014), 894-922.

Steiner, V. and Wrohlich, K., 'Household Taxation, Income Splitting and Labor Supply Incentives: A Microsimulation Study for Germany', CESifo Econ Stud 50 (2004), 541-68.

Steiner, V. and Wrohlich, K., 'Work Incentives and Labor Supply Effects of the "Mini-Jobs Reform” in Germany', Empirica 32/1 (2005), 99-116.

Van Soest, A., 'Structural Models of Family Labor Supply: A Discrete Choice Approach', Journal of Human Resources 30/1 (1995) 63-88.

Wagner, G. G., Frick, J. R. and Schupp, J., 'The German Socio-Economic Panel Study (SOEP): Scope, Evolution and Enhancements', Schmollers Jahrbuch 127/1 (2007), 139-69. 